\title{
Self-Enrichment of Merging Stellar Clusters
}

\author{
Natalie R. Hinkel*, Evan Scannapieco, F.X. Timmes ${ }^{\dagger}$ \\ Arizona State University, Joint Institute for Nuclear Astrophysics \\ E-mail: natalie.hinkeleasu.edu
}

We examine the mass-metallicity trend of nearby $z \sim 0.1$ galaxies presented by Tremonti et al. (2004) and compare it to detailed chemical evolution models. Our study was done in order to identify the environmental factors that govern observed metallicities. We found that the Tremonti data might not have been coeval, which would lead to a degeneracy between metallicity and age. While metallicity was measured using oxygen lines, we found that other metals offer different evolutions and therefore, various predictions for a mass-metallicity relationship. We also present initial studies investigating how short stellar lifetimes and metals ejected by massive stars influenced the observed chemical abundance variations in $10^{4}-10^{6} M_{\odot}$ galaxies. We will incorporate all of these factors into a hydrodynamic model to simulate the recent merger history of nearby galaxies.

10th Symposium on Nuclei in the Cosmos

July 27 - August 1, 2008

Mackinac Island, Michigan, USA

\footnotetext{
* Speaker.

†We would like to thank ASU's Fulton High Performance Computing Initiative for the use of their resources, including the SAGUARO cluster and Helios via FLASH. We are grateful to Christy Tremonti for her help and insight.
} 


\section{Introduction}

Chemical evolution is a broad field that spans both stellar and galactic scales. In 2004, Tremonti et al. (hereafter T04) found a strong mass-metallicity trend in a sample of $\sim 57,000$ galaxies, taken by the Sloan Digital Sky Survey (SDSS) Data Release 4 (DR4) (reproduced here in Fig. 1). Traditionally, heavy element outflows and stellar lifetime approximations have been utilized to explain this relationship. However, the spatial resolution now possible in more sophisticated numerical simulations can capture the observed dynamics and chemistry in galaxy. Therefore, we have analyzed the T04 mass-metallicity trend by allowing for inflows only and finite stellar lifetimes.

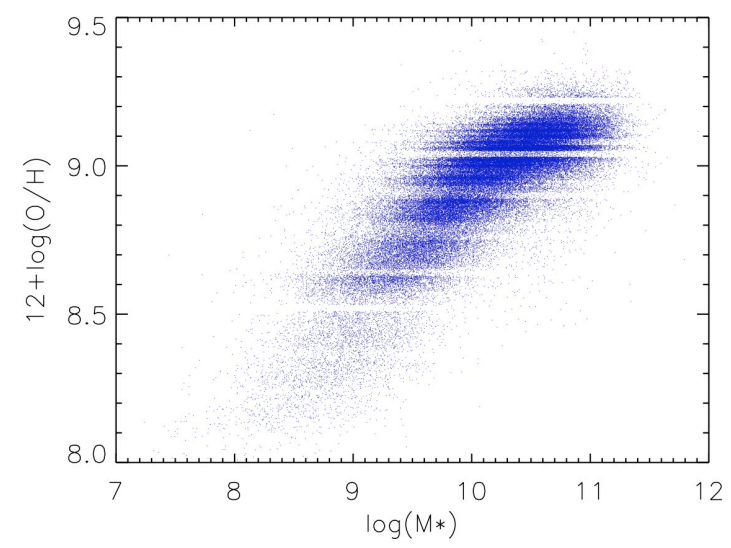

Figure 1: Reproduction of the T04 figure showing the relationship between stellar masses $\left(M_{\odot}\right)$ and the scaled gas-phase oxygen-abundance, or metallicity, for $\sim 57,000$ galaxies from SDSS DR4.

\section{Age-Metallicity Degeneracy}

A complication when looking at any mass-metallicity diagram is the degeneracy between age and metallicity because rapid stellar processes in massive galaxies mimic the metallicity in older galaxies. We have used two filters from the T04 SDSS data in order to determine a color vs. metallicity relation (Fig. 2). Because color is a measure of temperature and hence age,

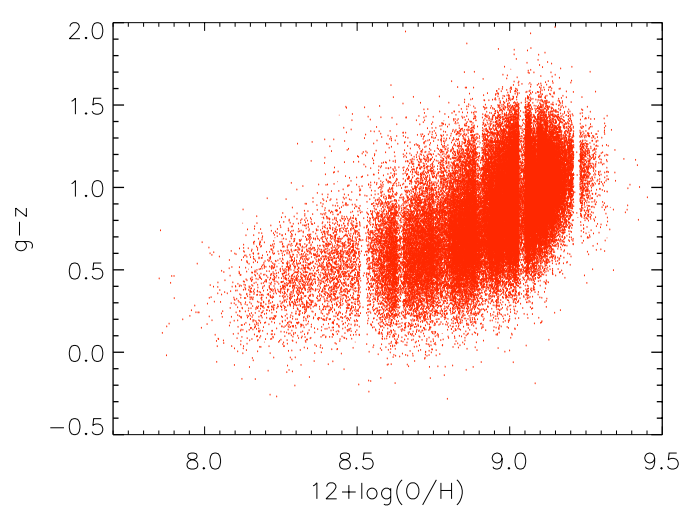

Figure 2: Color-mass plot illuminating the agemetallicity degeneracy. we would expect all points in this plot to be relatively concentrated in the y-direction if the galaxies were coeval. If there is degeneracy between age and metallicity, such that age distinctions were not sufficiently accounted for in this sample, then we would expect a linear trend indicating that younger galaxies had fewer metals and older galaxies had more. While there is a noteable linear and extended trend in Fig. 2, signaling some age variation in the sample, the most important feature is the concentrated area that is not completely linear. Even Malmquist bias, which populates one end more heavily than the other, cannot account for the curvature seen around $12+\log (O / H) \sim 9$. Therefore, while we feel that not all of the $\sim 57,000$ galaxies were coeval, a substantial portion might be the same age. To determine if this subsection would 
also produce the strong mass-metallicity trend, we are currently working with Tremonti on making more cuts to her data set in order to ensure galaxies are of the same age.

\section{Star Formation Rates}

Beginning with a simple model (Timmes et al. 1995, hereafter T95), the rate of change for the surface mass density of the interstellar medium per isotope was calculated radially using a zonetype model (Talbot \& Arnett 1971). We simulated an empty box at $t=0$ then matter, with the same composition as the Big Bang, was accreted over a period of $t=13.7$ Gyr. Mass in the gas-phase of the system was increased by two mechanisms: gas expelled after the death of a star and isotopes accreted from an outside source. The interstellar mass was decreased by isotopes trapped within a star during the lifetime of that star. The calculation ultimately relied on the normalized initial mass function (IMF), $\psi$, stellar birth rate, $B(t)=v\left(M_{\text {gas }} / M_{t o t}\right)^{2}$, the birthrate efficiency, $v$, and timescale for the galactic disk formation, $\tau_{\text {disk }}$. The standard values for the latter two were chosen based on observation: $v=2.8$ (Anders $\&$ Grevesse 1989) and $\tau_{\text {disk }}=4$ Gyr (Larson 1976). Thus, by varying the above parameters with the prescriptions described in T95 - stellar birth, accreted infall, Type II nucleosynthesis, Type Ia supernova, and low-intermediate stellar nucleosynthesis were modeled to reproduce the trends seen by $\mathrm{T} 04$.

Figure 4 was modeled using a simple Schmidt star formation rate (SFR)

$$
B(t)=v \sigma_{t o t}\left[\frac{\sigma_{g a s}(t)}{\sigma_{t o t}(t)}\right]^{n} M_{\odot} p c^{-2} G y r^{-1}
$$

where $\sigma_{t o t}$ and $\sigma_{\text {gas }}$ are the surface mass density for the total system and gas, respectively. However, other prescriptions for stellar formation that are equally viable. We show multiple SFRs in Fig. 3 and how they regulate the metallicity of the system as it evolves. The two lines in blue are representative of the Schmidt function (see figure caption). The red line is a Kennicutt step-function whose birthrate is zero until a critical mass of $7.5 M_{\odot} / p c^{2}$, or $10 \%$ of the solar neighborhood surface density, is reached at which point $n=2$. The green and black lines were modeled following the methods used by Daigne et al (2006). We constructed the birthrate using an exponential, timedependent SFR and an mass-dependent IMF:

$$
B=\psi(t) * \xi(\sigma)=v_{1} \exp \left[-1\left(t-t_{\text {init }}\right) / \tau_{1}\right] * c M_{\text {seed }}\left(\frac{\sigma_{g}}{\sigma_{\text {tot }}}\right)^{-(1+x)} M_{\odot} p c^{-2} G y r^{-1} .
$$

Like the authors, who found that the "best-fit model for normal star formation" was obtained with a minimum dark matter halo of $10^{7} M_{\odot}$, we adopted $v_{1}=0.2, \tau_{1}=2.8$, and $t_{\text {init }}=0.102$ for $z_{\text {init }}=30$, in accordance with their cosmological model (see eq. 3 of Daigne et al. 2006). The IMFs were Salpeter and Scalo, with $x=1.3$ (green) and 1.7 (black), respectively, and the constant $c$ was a normalization term specific to each IMF. However, because Daigne et al. initialized their model with a burst of massive star formation, which eventually led to the "normal star formation" described by their model above, we found it necessary to add a seed of mass such that $M_{\text {seed }}=$ $10^{2} M_{\odot}$, since we were only looking at the epoch of "normal star formation." Because the T04 data only represents one point on this time evolution graph, at $t=13.7 \mathrm{Gyr}$, any of the SFRs can be scaled up or down in order to match the existing data. Future data at a further redshift would yield 
a better determination of the appropriate SFR, by matching the metallicity trend observed to one of the models here.

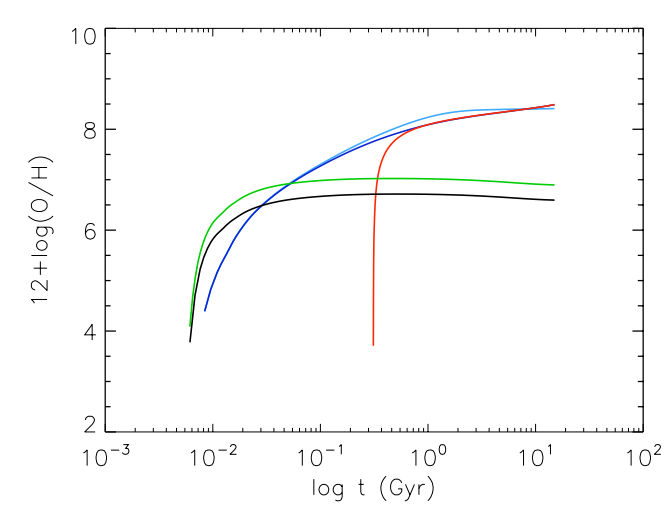

Figure 3: Various SFRs versus metallicity for the Schmidt function ( $n=1,2-$ light/dark blue, Eq. 3.1), Kennicutt step-function (red), and Daigne et al. (2006) model in green/black (Eq. 3.2).

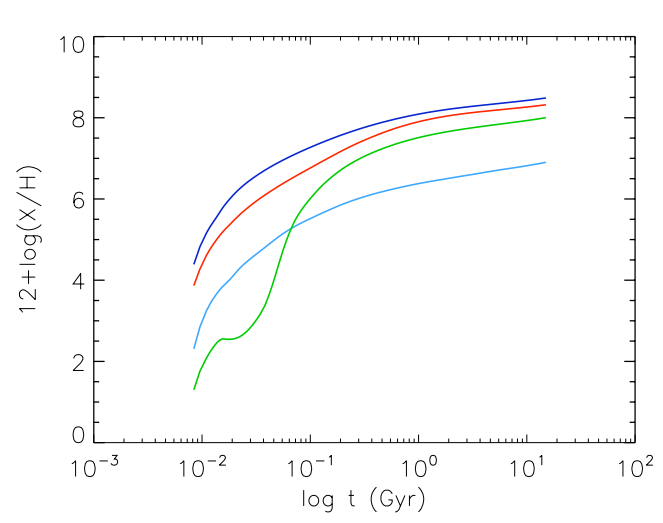

Figure 4: Heavy metallicity evolution using $100 M_{\odot}$ as the initial solar neighborhood surface density and a Schmidt $(n=2)$ SFR. Plotted are C (red), N (dark green), $\mathrm{O}$ (dark blue), and Fe (light blue).

\section{Alternative Metallicities}

All of the metallicity measurements done by T04 were done with respect to oxygen. However, while oxygen lines are easy to observe, we questioned whether it was representative of the other metals. Therefore, we examined the evolution of four metals $(\mathrm{C}, \mathrm{N}, \mathrm{O}, \& \mathrm{Fe})$ with time, as plotted in Fig. 4. It is clear from the diagram that different elemental ratios give different signature, particularly nitrogen (green). While carbon, oxygen, and iron are produced in higher-mass stars $\left(>8 M_{\odot}\right)$, nitrogen comes from intermediate-mass stars $\left(<8 M_{\odot}\right)$. When intermediate-mass stars die, they inject nitrogen back into the interstellar medium, however, because intermediatemass stars live longer, the injection happens later. And, because there is a higher population of intermediate-mass stars than higher-mass stars, the injection is much stronger. Thus, because of the different evolution that nitrogen has over the other metals, it gives a varying prediction for the mass-metallicity relationship of galaxies.

\section{Conclusions and Future Work}

The T04 data has been highly analyzed and many attempts have been made to reproduce the mass-metallicity trend line observed. One of the more significant findings from our study is the possibility that the $\sim 57,000$ galaxies, while all at $z \sim 0.1$, might not all be coeval. Our other results suggest that current simulations have enough sophistication and fidelity to the ongoing physics to closely reproduce the elemental abundances observed. It would be interesting if similar data sets were able to offer abundance information at further redshifts and with multiple metals. The next step that we plan on taking with our model, in order to motivate the T04 data (here Fig. 1), is to extend the simulation from one-zone to multiple zones. 
While analyzing the data presented by T04, we have also begun working on simulating stellar clusters using a 3D hydrodynamic simulation, namely FLASH. The FLASH code is modular, scalable, features adaptive mesh refinement, self-gravity, and has the ability to run in parallel. U1timately, it is with this tool that we will begin modeling the history of stellar clusters, as shown in Fig. 5. By creating 3D hydrodynamic models
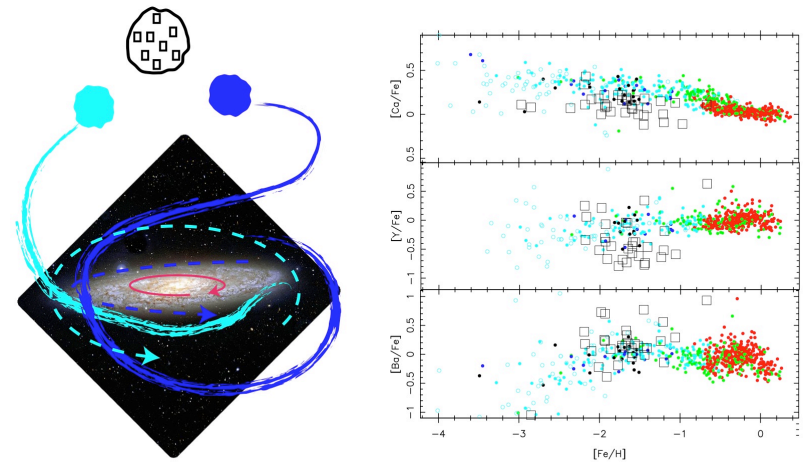

Figure 5: Illustration of merging history (here NGC 253 - CFHT). The blue satellite galaxies, after merging with our galaxy in the past, have dispersed their stellar content in the halo. The black dwarf contains the signature of additional, undisturbed chemical evolution. of $10^{4}-10^{6} M_{\odot}$ mass-scale stellar clusters, it is possible to track the evolution of the system's kinematics and chemical abundances. The goal is to assess how inhomogeneities imparted by shorter stellar lifetimes and nucleosynthesis by massive stars influence the observed variation abundances within a stellar cluster. These models will then be compared with observations of metal-poor stars in the Galactic halo and massive star forming regions in the Local Group. By facilitating new, testable predictions on recent merger activity, these efforts will take chemical enrichment simulations at the cluster-scale to an unprecedented level. Carollo et al. (2007) recently found evidence that the Galaxy has both an inner net prograde halo and an outer net retrograde halo. This suggests that if the Galaxy has had recent interactions with the Local Group, we would be able to unravel their sequence from numerical simulations of the the chemical makeup of the galaxies.

\section{References}

[1] Anders, E., \& Grevesse, N., Abundances of the Elements - Meteoritic and Solar, Geochim. Cosmochim. Acta. 53 (1989) 197.

[2] Carollo, D. et al. Two Stellar Components in the Halo of the Milky Way, Nature 450 (2007) 1020.

[3] Daigne, F. et al. Hierarchical Growth and Cosmic Star Formation: Enrichment, Outflows, and Supernova Rates, ApJ 647 (2007) 1020.

[4] Larson, R. B. Models for the Formation of Disc Galaxies, MNRAS 176 (1976) 31.

[5] Salpeter, E. E. The Luminosity Function and Stellar Evolution, ApJ 121 (1955) 161.

[6] Scalo, J. M. The Stellar Initial Mass Function, Fund. Cosmic. Phys. 11 (1986) 1.

[7] Schmidt, M. The Rate of Star Formation, ApJ 129 (1959) 243.

[8] Talbot, J.R., Jr.. \& Arnett, W.D. The Evolution of Galaxies. I. Formulation and Mathematical Behavior of the One-Zone Model, ApJ 170 (1971) 409.

[9] Timmes, F.X. et al. (T95) Galactic Chemical Evolution: Hydrogen through Zinc, ApJ 98 (1995) 617.

[10] Tremonti, C.A., et al. (T04) The Origin of the Mass-Metallicity Relation: Insights from 53,000 Star-Forming Galaxies in the Sloan Digital Sky Survey, ApJ 613 (2004) 898. 